\title{
Relationship Among Tumor Attenuation Value of Pre-contrast Computed Tomography (CT), Washout Rate and Constituent Cells in Adrenal Adenoma: Proposition of a New Approach for Diagnosing Adrenal Adenoma on Dynamic CT
}

\author{
AKIHIRO NISHIE $^{1}$, MASAAKI SUGIMOTO $^{2}$, YOSHIKI ASAYAMA ${ }^{1}$, KOUSEI ISHIGAMI $^{1}$, \\ YASUHIRO USHIJIMA ${ }^{1}$, YUKIHISA TAKAYAMA ${ }^{1}$, DAISUKE OKAMOTO ${ }^{1}$, NOBUHIRO FUJITA ${ }^{1}$, \\ AKIRA YOKOMIZO $^{3}$, KATSUNORI TATSUGAMI $^{3}$ and HIROSHI HONDA ${ }^{1}$ \\ Departments of ${ }^{1}$ Clinical Radiology, ${ }^{2}$ Anatomic Pathology and ${ }^{3}$ Urology, \\ Graduate School of Medical Sciences, Kyushu University, Fukuoka, Japan
}

\begin{abstract}
Aim: To elucidate the relationship among tumor attenuation of pre-contrast-enhanced (TApre) computed tomography $(C T)$, washout rate and clear cell ratio $(C C R)$ in adrenal adenoma (AA) and propose a new approach for diagnosing AA on dynamic CT. Materials and Methods: The training set consisted of $43 A A s$ and 15 non-AAs, while the validation set comprised $44 A A s$ and 11 non-AAs. Using the training set, the pairwise correlation between CCR, TApre and washout rate in $A A$ was evaluated by linear regression analysis. A predictive formula for diagnosing $A A$ was established by multiple logistic regression analysis using washout rate and TApre. Using the validation set, the diagnostic performance of this formula was investigated by comparing with the conventional diagnostic criteria: TApre $\leq 10 \mathrm{HU}$ or washout rate $\geq$ an optimal threshold calculated in the training set. Results: Washout rate increased as CCR decreased, and as TApre increased. The formula predicting the probability of $A A$ was: $p(A A)=1 /\{1+\exp (-1.5257+0.4923 \times$ TApre-0.3264Xwashout rate)\}. Diagnostic performance of this formula was sensitivity of $93.2 \%$ and accuracy of $89.1 \%$, while for the conventional diagnostic criteria, sensitivity was 81.8-86.4\% and accuracy 81.8-83.6\%. Conclusion: The diagnostic potential of dynamic CT for diagnosing AA may be improved by changing the threshold of washout rate based on substituting TApre for CCR.
\end{abstract}

Correspondence to: Akihiro Nishie, MD, Department of Clinical Radiology, Graduate School of Medical Sciences, Kyushu University, 3-1-1, Maidashi, Higashi-ku, Fukuoka, 812-8582, Japan. Tel: $\quad+81$ 926425695, Fax: +81 926425708, e-mail: anishie@radiol.med.kyushu-u.ac.jp

Key Words: CT, adrenal adenoma, clear cell, compact cell, washout.
Adrenal adenomas (AAs) are the most common adrenal lesions, found in $3 \%$ of cases at autopsy (1). Therefore, it is critical to differentiate them from other adrenal lesions in daily practice. AA is composed histologically of both clear cells and compact cells (2). Yamada et al. (3) suggested that the low tumor attenuation on pre-contrast computed tomographic (CT) imaging in AA represents the abundant intracytoplasmic lipids in clear cells. Therefore, AA is often classified into lipid-rich and lipid-poor AA based on the tumor attenuation value of the pre-contrast-enhanced CT image. An adrenal tumor with attenuation of $10 \mathrm{HU}$ or less on pre-contrast-enhanced CT is likely to be a lipid-rich AA (4). Chemical-shift magnetic resonance imaging (MRI) may be more useful in diagnosing a lesion as AA because this sequence is more sensitive to small amounts of fat than precontrast-enhanced CT (5). However, lipid-poor AA cannot be diagnosed using these two methods. In such cases, the washout rate after contrast enhancement can be used.

AA is known to show a high washout rate after contrast enhancement on dynamic CT (2). This tumor can be differentiated from pheochromocytoma, adrenal metastasis and adrenal cancer, which reportedly show low washout rates (2, 6-8). However, there is an overlap in washout behavior between pheophromocytoma and AA (9). Previous reports have shown that lipid-rich AAs do not demonstrate washout patterns different from those of lipid-poor AAs $(4,8,10)$. On the other hand, although adrenal hyperplasia closely resembles AA in histology and is composed of both clear cells and compact cells, the variability in washout rates has been reported to be associated with differences in constituent cells of adrenal hyperplasia (11). That is, the washout rate of adrenal hyperplasia increases as the clear cell ratio (CCR) decreases or as tumor attenuation value of the pre-contrastenhanced CT image (TApre) increases. We hypothesized that 
the washout rate of AA would also depend on CCR or TApre and that it should be possible to differentiate AA from other adrenal tumors more clearly by changing the diagnostic threshold of washout rate based on CCR or TApre.

The purpose of this study was to elucidate the relationship among the tumor attenuation values of pre-contrast-enhanced $\mathrm{CT}$, washout rate and constituent cells in AA and to propose a new approach for diagnosing AA, aiming for better diagnostic performance on dynamic CT.

\section{Materials and Methods}

Patients. This study was approved by the Ethical Committee on clinical study at our institution, which conforms to the provisions of the Declaration of Helsinki. Institutional Review Board approval (no. 25-45) was obtained, and the requirements for informed consent were waived for this retrospective study. Referring to the medical data recorded at our hospital, 123 consecutive patients who underwent a preoperative CT examination and adrenal resection between October 2001 and June 2015 were selected. Initially, four patients were excluded due to differences in CT protocol; then a further six patients were excluded because hematoxylin-eosinstained tumor slides were not available. As a result, 113 patients were finally enrolled in this study. The CT examinations were performed within 3 months before surgery for all patients. The patients consisted of 46 men and 67 women (age range=19-81 years; mean age $=53$ years). The number of nodular lesions evaluated was 113 (one in each patient). Forty-seven lesions were located in the right adrenal gland and sixty-six lesions in the left adrenal gland. Their diameter range was 1.1 to $11.5 \mathrm{~cm}$, and the mean diameter was $2.8 \mathrm{~cm}$. The patients were divided into the following two groups: Training group: A total of 58 consecutive patients between October 2001 and June 2010; and the validation group, comprising 55 consecutive patients between July 2010 and June 2015. The tumor details of each group are shown in Table I. The average size of lesions in the training set was $2.9 \mathrm{~cm}$ (range $=1.2-11.5 \mathrm{~cm}$ ), while that of the validation set was $2.6 \mathrm{~cm}$ (range=1.1-8.0 cm). The training set was utilized to analyze radiological and pathological parameters in AA and to establish a predictive formula for differentiating AA from other adrenal tumors, while the validation set was used to evaluate the diagnostic performance of the predictive formula in a different patient population.

CT protocol. Contrast-enhanced multi-phase helical CT was performed with a 4- or 64-multidetector row computed tomography (MDCT) scanner (Aquilion; Toshiba Medical, Tokyo, Japan) in all patients. For the first CT protocol, the scanning parameters with the 64-MDCT were $120 \mathrm{kVp}$, automatically set mAs values, 1-mm collimation reconstructed to a slice thickness of $5 \mathrm{~mm}$, and a pitch of 27 , while the scanning parameters with the 4-MDCT were $120 \mathrm{kVp}, 300 \mathrm{mAs}, 3-\mathrm{mm}$ collimation reconstructed to a slice thickness of $5 \mathrm{~mm}$, and pitch of 3 . The images were obtained in the craniocaudal direction. Firstly, pre-contrast CT images were scanned. A total of $100 \mathrm{ml}$ of a nonionic iodinated contrast agent (iopamidol: Iopamiron 370; Bayer, Osaka, Japan) was injected through the antecubital vein for $40 \mathrm{~s}$ at an injection rate of $2.5 \mathrm{ml} / \mathrm{s}$ using a power injector. Early and delayed phases were scanned 60 and $240 \mathrm{~s}$ after the contrast injection.
The second CT protocol was performed with the same 64-slice MDCT scanner with a gantry rotation time of $0.5 \mathrm{~s}$. Firstly, precontrast $\mathrm{CT}$ images were scanned. Intravenous nonionic contrast material (1.62 mI/kg, max $100 \mathrm{mI})$ containing $370 \mathrm{mg}$ iodine $/ \mathrm{ml}$ (Iopamiron 370; Bayer Schering Pharma) was injected by means of an automated power injector, and the injection time was $30 \mathrm{~s}$. The scan parameters were as follows: section collimation, $0.5 \mathrm{~mm}$; helical pitch, 53; auto $\mathrm{mA}$; and $120 \mathrm{kVp}$. The bolus tracking technique was used to determine the timing of the dynamic study; the arterial phase was obtained during a single breath-hold $15 \mathrm{~s}$ after a 100-HU elevation of density in the descending aorta. The early and delayed phases were acquired at 60 and $240 \mathrm{~s}$, respectively, after the initiation of contrast material injection. The section thickness and reconstruction interval were 1.0 and $3.0 \mathrm{~mm}$, respectively.

Imaging interpretation. For the training set, two abdominal radiologists (Y.U. and Y.T.) attempted to localize all 58 adrenal lesions on the pre-contrast image, and early and delayed phases, based on the operation record. The two radiologists examined CT attenuation values of adrenal lesions on each phase quantitatively. The slice on which the adrenal lesion appeared the largest was selected, and the tumor attenuation value was measured by placing a region of interest (ROI) that was as large as possible in a consensus fashion. An ROI of the same size was placed at the same position of the tumor on an image of each phase. TApre, TAearly and TAdelay were defined as tumor attenuation values of precontrast, early and delayed phases, respectively. The absolute percentage washout (APW) of each adrenal lesion was calculated using the following formula: APW $(\%)=($ TAearly - TAdelay $) /$ (TAearly - TApre) $\times 100$.

For the validation set, two different abdominal radiologists (D.O. and N.F.; Readers 1 and 2) attempted to localize all 55 adrenal lesions on pre-contrast image, and early and delayed phases, based on the operation record. The two radiologists independently examined CT attenuation values of adrenal lesions on each phase with the same method as described above. The APW of each adrenal lesion was also calculated.

Pathological analysis. One pathologist (M. S.), who was unaware of the imaging data, reviewed the hematoxylin-eosin stained tumor slides of 43 AAs in the training set. He referred to the whole slides and qualitatively assessed the CCR for each AA at 10-fold magnification. This ratio was recorded as rounded to the nearest $5 \%$.

Statistical analysis. For 43 AAs of the training set, linear regression analysis was performed between each pair of the three radiological and pathological parameters: namely, CCR, TApre and APW. When there was significant correlation between APW and TApre (or CCR), multiple logistic regression analysis was performed to establish a predictive formula for diagnosing AA using all 58 adrenal lesions of the training set. When the probability of AA obtained by the formula was 0.5 or more, the adrenal tumor was judged as being AA. The diagnostic performance of this predictive formula for AA in the training group was compared with those of three diagnostic criteria (DC) of AA as follows: DC1: TApre $\leq 10 \mathrm{HU}$ (4); DC2: APW at or exceeding the threshold calculated using receiver operating characteristic (ROC) analysis; and DC3: TApre $\leq 10 \mathrm{HU}$ or APW at or exceeding the ROC threshold. The significance of differences in sensitivity, specificity and accuracy 
Table I. Tumor profile of the training and validation sets.

\begin{tabular}{lcc}
\hline Adrenal lesion & Training set, $\mathrm{n}$ & Validation set, $\mathrm{n}$ \\
\hline Cortical adenoma & 43 & 44 \\
Pheochromocytoma & 12 & 5 \\
Adrenal metastasis & 1 & 2 \\
Adrenocortical carcinoma & 1 & 2 \\
Cryptococcosis & 1 & 0 \\
Malignant lymphoma & 0 & 1 \\
Myelolipoma & 0 & 1 \\
\hline Total & 58 & 55 \\
\hline
\end{tabular}

The number of cases is reported.

Table II. Correlation between clear cell ratio (CCR), tumor attenuation value of pre-contrast (TApre) and absolute percentage washout (APW).

\begin{tabular}{lccc}
\hline & CCR & TApre & APW \\
\hline CCR & - & -0.54 & -0.31 \\
TApre & $p<0.001$ & - & 0.37 \\
APW & $p=0.026$ & $p<0.01$ & - \\
\hline
\end{tabular}

TAearly and TAdelay: Tumor attenuation values of early and delayed phases, respectively. APW of each nodular lesion $(\%)=($ TAearly TAdelay)/(TAearly - TApre $) \times 100$. The upper right data are correlation coefficients. The lower left data represent statistical significance.

Table III. Diagnostic performance of the predictive formula and the other three diagnostic criteria (DC) of adrenal adenoma in the training set.

\begin{tabular}{llcl}
\hline & Sensitivity & Specificity & Accuracy \\
\hline Predictive formula & $93.0 \%(40 / 43)$ & $86.7 \%(13 / 15)$ & $91.4 \%(53 / 58)$ \\
DC1 & $60.5 \%(26 / 43) *$ & $100.0 \%(15 / 15)$ & $70.7 \%(41 / 58)^{*}$ \\
DC2 & $67.4 \%(29 / 43) *$ & $66.7 \%(10 / 15)$ & $67.2 \%(39 / 58)^{*}$ \\
DC3 & $95.3 \%(41 / 43)$ & $66.7 \%(10 / 15)$ & $87.9 \%(51 / 58)$ \\
\hline
\end{tabular}

DC1: TApre $\leq 10$ HU DC2: APW $\geq 51.4 \%$; DC3: TApre $\leq 10$ HU or APW $\geq 51.4 \%$. The values given in parentheses are the number of tumors correctly diagnosed out of the total number of target tumors. *Significantly different from the predictive formula at $p<0.01$.

between the predictive formula and each of these three DCs were evaluated by the McNemar test.

Furthermore, the diagnostic performance of this predictive formula in the validation set, which was a different patient population, was evaluated using the two radiologists' data measured independently. It was also compared with those of the three DCs of AA as described above. The significance of differences in sensitivity, specificity and accuracy between the predictive formula and each of the three DCs was again evaluated by the McNemar test. $p$-Values less than 0.05 were considered statistically significant.

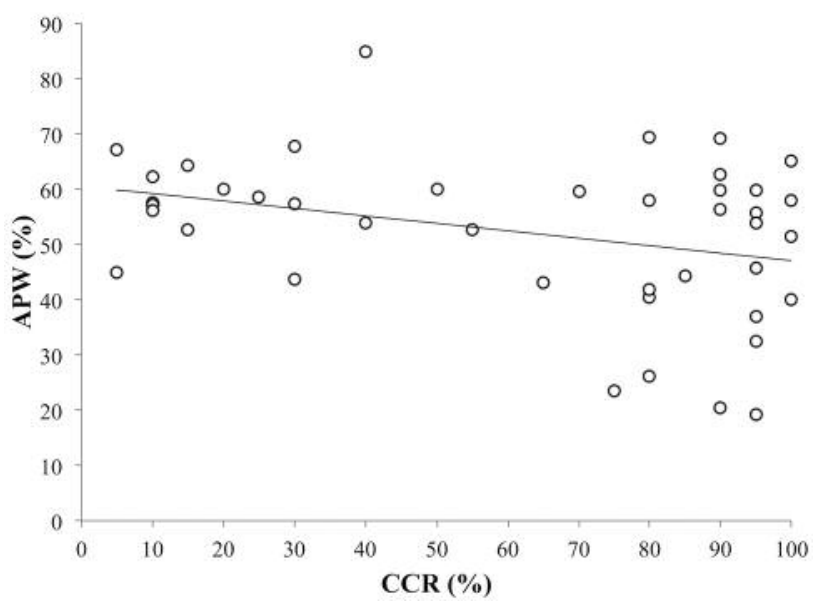

Figure 1. Graph showing a significant correlation between the clear cell ratio (CCR) and absolute percentage washout (APW) based on the data of the training group. Correlation coefficient was -0.31. APW (\%) $=-0.1351 \times C C R(\%)+60.59$.

\section{Results}

Correlation coefficients and statistical significance among CCR, TApre and APW are shown in Table II. There was a significant correlation between each pair. CCR decreased as TApre increased. APW increased as CCR decreased (Figure 1) and as TApre increased.

Because CCR cannot be assessed preoperatively, multiple logistic regression analysis was performed using APW and TApre. The formula predicting the probability of AA was: $\mathrm{p}(\mathrm{AA})=1 /\{1+\exp (-1.5257+0.4923 \times$ TApre $-0.3264 \times$ APW $)\}$. The diagnostic performance of the predictive formula for diagnosing AA in the training group was as follows: sensitivity $93.0 \%$ (40/43), specificity $86.7 \%(13 / 15)$, and accuracy $91.4 \%$ (53/58). The optimal threshold of APW for diagnosing AAs was found to be $51.4 \%$ when calculated using ROC analysis. The Az value of the ROC curve was 0.71. Diagnostic performance of the three DCs of AA together with that of the predictive formula are shown in Table III. There was a significant difference in sensitivity and accuracy between the predictive formula and DC1 and DC2 $(p<0.01)$. No significant difference in specificity between the predictive formula and DC1 or DC2 was obtained. Although there was no significant difference in sensitivity, specificity or accuracy between the predictive formula and DC3, the accuracy of the predictive formula (91.4\%) was slightly higher than that of DC3 (87.9\%). Representative cases are shown in Figures 2-4.

Diagnostic performance of the three DCs of AA including that of the predictive formula are shown in Tables IV (Reader 1) and V (Reader 2). For both readers, there was a significant difference in sensitivity and accuracy between the 

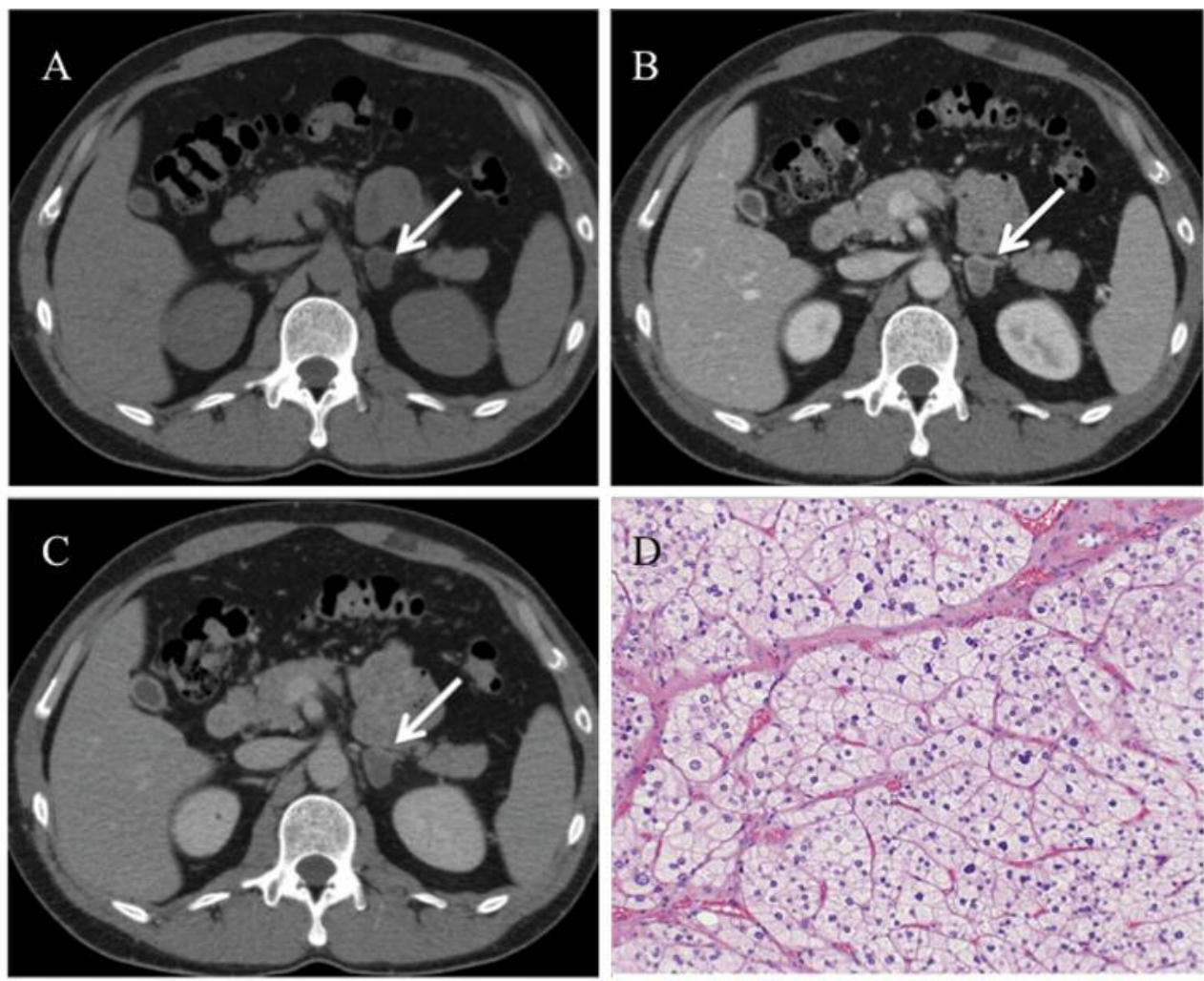

Figure 2. A 35-year-old man with a history of primary aldosteronism. A-C: Pre-contrast-enhanced computed tomographic (CT) image, and earlyand delayed-phase images, respectively. A 2.1-cm nodular lesion (adrenal adenoma) can be seen in the left adrenal gland on dynamic CT (arrows). Tumor attenuation value of pre-contrast (TApre), TAearly and TAdelay were-2, 68 and $36 \mathrm{HU}$, respectively. Absolute percentage washout was $45.7 \%$. D: The clear cell ratio was 95\%. Pathologically, fibrous septa were identified between clusters of clear cells. For comparison, the predictive formula made a correct diagnosis of adrenal adenoma. The probability of adrenal adenoma using the formula was 1.0000.

Table IV. Diagnostic performance of the predictive formula and the other three diagnostic criteria of adrenal adenoma in the validation set (image analysis by Reader 1).

\begin{tabular}{llll}
\hline & \multicolumn{1}{c}{ Sensitivity } & \multicolumn{1}{c}{ Specificity } & \multicolumn{1}{c}{ Accuracy } \\
\hline Predictive formula & $93.2 \%(41 / 44)$ & $72.7 \%(8 / 11)$ & $89.1 \%(49 / 55)$ \\
DC1 & $52.3 \%(23 / 44)^{*}$ & $90.9 \%(10 / 11)$ & $60.0 \%(33 / 55)^{*}$ \\
DC2 & $61.4 \%(27 / 44)^{*}$ & $81.8 \%(9 / 11)$ & $65.4 \%(36 / 55)^{*}$ \\
DC3 & $86.4 \%(38 / 44)$ & $72.7 \%(8 / 11)$ & $83.6 \%(46 / 55)$ \\
\hline
\end{tabular}

DC1: TApre $\leq 10$ HU DC2: APW $\geq 51.4 \%$; DC3: TApre $\leq 10$ HU or $\mathrm{APW} \geq 51.4 \%$. The values given in parentheses are the number of tumors correctly diagnosed out of the total number of target tumors. *Significantly different from the predictive formula at $p<0.01$.

predictive formula and DC1, and DC2 $(p<0.01)$. No significant difference in specificity between the predictive formula and DC1 or DC2 was obtained. Although there was no significant difference in sensitivity, specificity or accuracy between the predictive formula and DC3, the accuracy of the
Table V. Diagnostic performance of the predictive formula and the other three diagnostic criteria of adrenal adenoma in the validation set (image analysis by Reader 2).

\begin{tabular}{llll}
\hline & \multicolumn{1}{c}{ Sensitivity } & Specificity & \multicolumn{1}{c}{ Accuracy } \\
\hline Predictive formula & $93.2 \%(41 / 44)$ & $72.7 \%(8 / 11)$ & $89.1 \%(49 / 55)$ \\
DC1 & $45.5 \%(20 / 44)^{*}$ & $81.8 \%(9 / 11)$ & $52.7 \%(29 / 55)^{*}$ \\
DC2 & $65.9 \%(29 / 44)^{*}$ & $81.8 \%(9 / 11)$ & $69.1 \%(39 / 55)^{*}$ \\
DC3 & $81.8 \%(36 / 44)$ & $81.8 \%(9 / 11)$ & $81.8 \%(45 / 55)$ \\
\hline
\end{tabular}

DC1: TApre $\leq 10$ HU DC2: APW $\geq 51.4 \%$; DC3: TApre $\leq 10$ HU or $\mathrm{APW} \geq 51.4 \%$. The values given in parentheses are the number of tumors correctly diagnosed out of the total number of target tumors. *Significantly different from the predictive formula at $p<0.01$. The sensitivity of the predictive formula tended to be higher than that of DC3 $(p=0.073)$.

predictive formula (Reader 1, 89.1\%; Reader 2,89.1\%) was slightly higher than that of DC3 (Reader 1, 83.6\%; Reader $2,81.8 \%$ ). For Reader 2 , the sensitivity of the predictive formula (93.2\%) tended to be higher than that of DC3 $(81.8 \%)(p=0.073)$. 

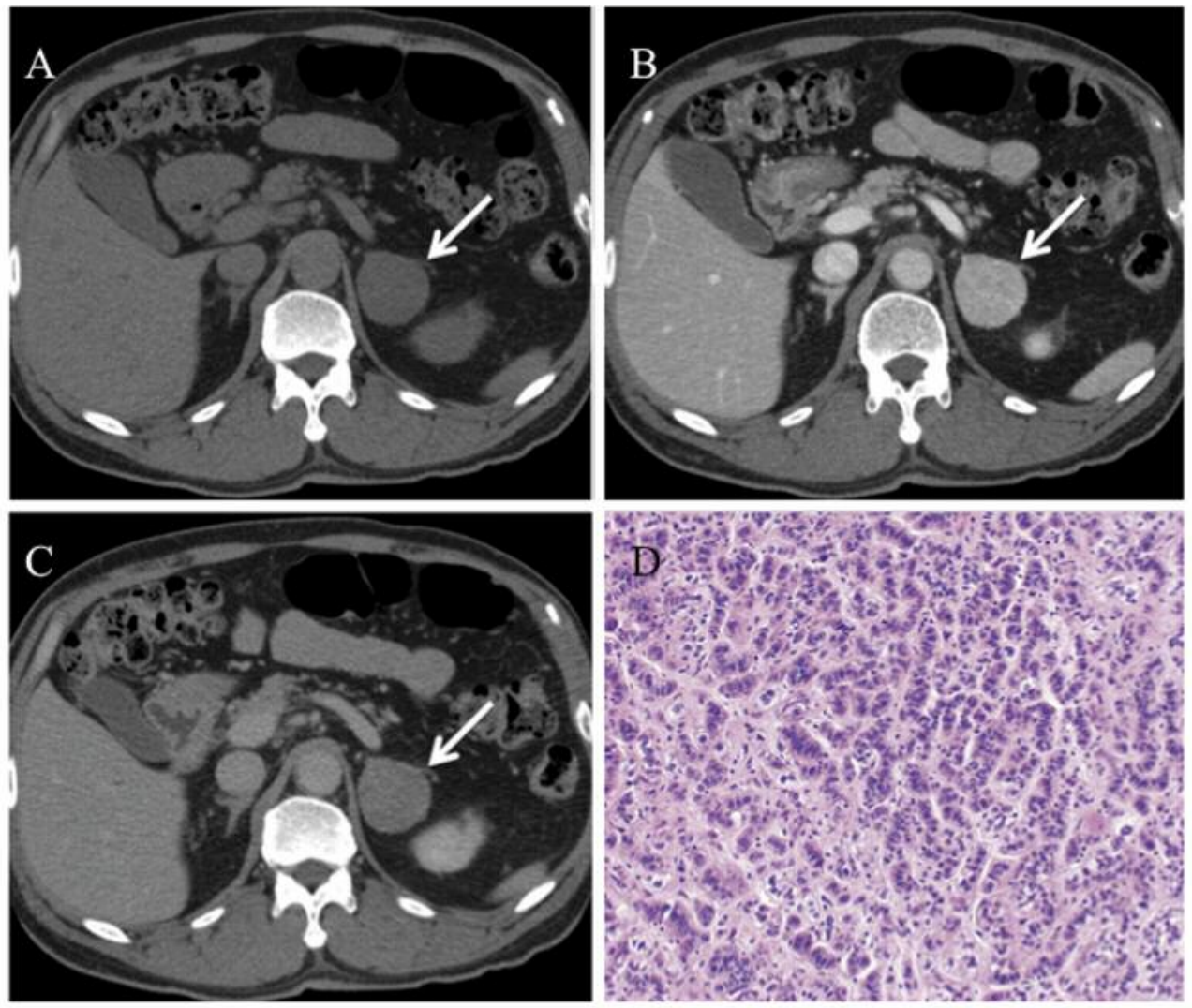

Figure 3. A 58-year-old man with a history of primary aldosteronism. A-C: Pre-contrast-enhanced computed tomographic (CT) image, and earlyand delayed-phase images, respectively. A 3.6-cm mass (adrenal adenoma) can be seen in the left adrenal gland on dynamic CT (arrows). Tumor attenuation value of pre-contrast (TApre), TAearly and TAdelay were 27, 152 and $68 \mathrm{HU}$, respectively. Absolute percentage washout was $67.2 \%$. D: The clear cell ratio was 5\%. Pathologically, fibrous septa were not identified in the mass, most of which was composed of compact cells. For comparison, the predictive formula made a correct diagnosis of adrenal adenoma. The probability of adrenal adenoma using the formula was 1.0000 .

\section{Discussion}

$\mathrm{CT}$ is the first-choice imaging modality for diagnosing adrenal tumors. An adrenal tumor with $\leq 10 \mathrm{HU}$ on precontrast CT is likely to be AA (4). Chemical-shift MRI may be more useful in diagnosing as AA because this sequence is more sensitive to small amounts of fat than pre-contrast CT (5). However, lipid-poor AA cannot be diagnosed using these two methods. In such cases, the washout rate after contrast enhancement can be used. On dynamic contrast-enhanced imaging, most AAs have a strong enhancing effect in the early phase and are less enhanced in the delayed phase (2). Pheochromocytomas and adrenocortical adenocarcinomas are enhanced strongly in the early phase but are continuously enhanced through the delayed phase (1, 12-16). Many adrenal metastases may show a low washout rate (17), although it depends on the kind of primary tumor. As a result, the washout rate of AA should be the largest. However, there is some degree of overlap in washout rates between AA and other adrenal tumors (9).
In this study, significant correlations were obtained among CCR, TApre and APW in AA. The correlation between CCR and TApre has been reported previously in AA (3), with the authors suggesting that low TApre represents abundant intracytoplasmic lipid in clear cells. The same conclusion could be drawn in this study. On the other hand, APW was significantly correlated with CCR and TApre. That is, APW increased as CCR decreased, and as TApre increased. Fibrous septa containing blood vessels are known to be present between nests and cords of clear cells in AA (18). We consider that the fibrous septa between clear cells, which are not observed between compact cells, could account for the difference in APW. A stasis of contrast medium may occur in the delayed phase in a tumor mainly composed of clear cells in comparison with a compact cell-rich tumor. Previous reports have shown that lipid-rich AAs do not demonstrate washout patterns different from those of lipidpoor AAs $(8,10)$. However, our pathologicoradiological investigation demonstrated that lipid-rich AAs exhibit washout patterns different from those of lipid-poor AAs. 
Therefore, we consider that AA should be diagnosed based on the washout rate compensated with CCR and that TApre should be substituted for CCR because there we found significant correlation between TApre and CCR. Unfortunately, CCR can be evaluated only post-operatively. In other words, the threshold of the washout rate for diagnosing AA should be changed according to TApre being substituted for CCR. As a result, a predictive formula for AA was newly created here. Such a diagnostic approach could be useful in resolving overlaps in the washout rate between different adrenal tumors.

The predictive formula for AA showed higher diagnostic performance in comparison with the other three diagnostic criteria (DCs 1-3). Our predictive formula was slightly superior to DC3, which is generally used to diagnose AA, although the difference in accuracy was not statistically significant. As seen in Figure 4, AA not showing TApre $\leq 10$ HU or APW $\geq 51.4 \%$ can be correctly diagnosed using this formula. Similar results were obtained for different patient populations (validation set) and readers (Readers 1 and 2), suggesting that our predictive formula may be a reproducible and reliable method. Additionally, it is also a simple method because the probability of AA can be obtained by plugging two values (TApre and APW) into the equation saved in any spreadsheet software.

The scan timing of delayed phase is important in evaluating washout patterns. Although the validity of radiological parameters calculated using 10-minute or longer delayed enhanced attenuation has been reported $(4,6)$, a shorter delayed enhanced phase such as a 3- or 5-minute scan is also useful for the differentiation of AAs from non-AAs $(8,19)$. Therefore, we believe that our protocol including 4-minute delayed scanning may be acceptable for assessment of the washout pattern of adrenal lesions. Shorter delayed scanning also leads to improvement of the examination throughput in daily practice. According to the report of Korobkin et al. (4), the sensitivity and specificity for diagnosing AA based on APW using 10-minute delayed scan were $88 \%$ and $89 \%$, respectively (4). Diagnostic performance of our predictive formula was comparable to their result, showing slightly higher sensitivity and slightly lower specificity. By combining our new approach with 10-minute or longer delayed scanning, more precise diagnostic ability for AA might be obtained.

The present study has several limitations. Firstly, the number of non-AAs enrolled in this study was relatively small because only surgically resected adrenal tumors were collected. Most adrenal metastases might not be surgically resected, and many pheochromocytomas were pre-operatively evaluated on MRI. This tendency may lead to selection bias. Secondly, two kinds of CT protocol were used in this study. This is a problem that occurred accompanied with renewal of CT equipment at our institution because patients who underwent adrenal resection between October 2001 and June 2015 were included. However,
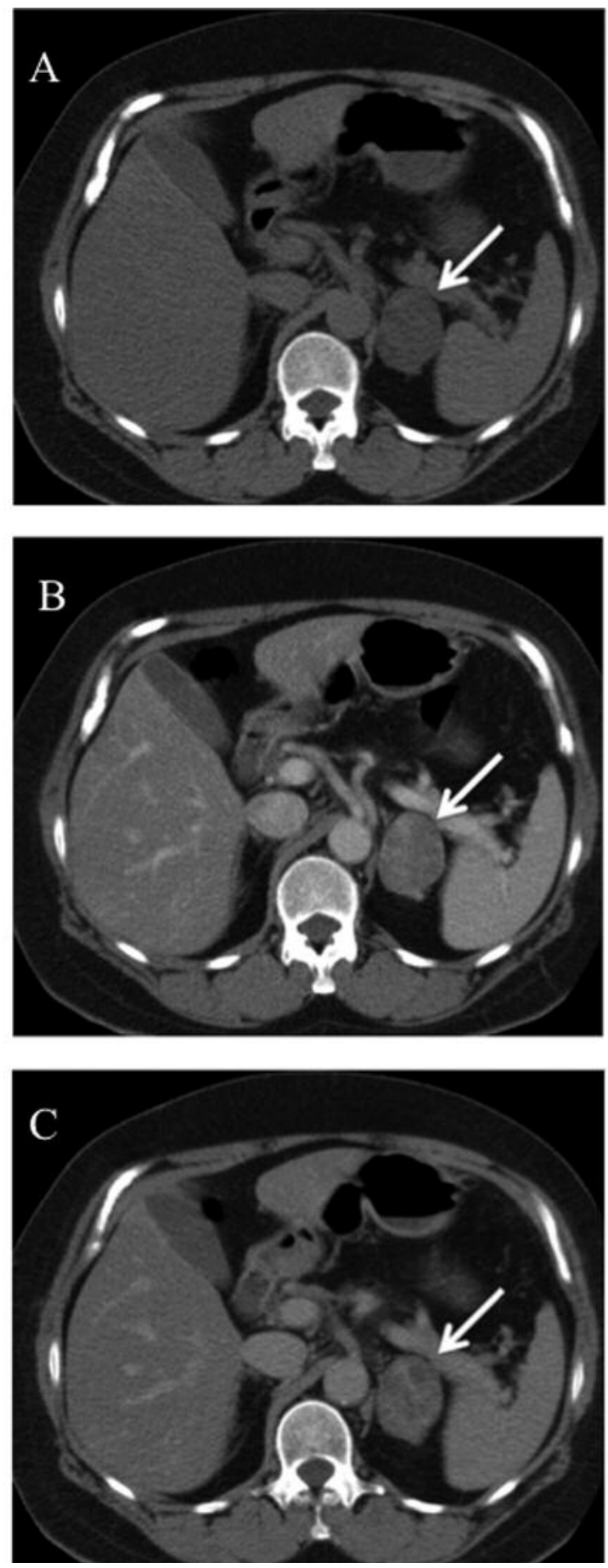

Figure 4. A 54-year-old woman with a history of preclinical Cushing's syndrome. A-C: Pre-contrast-enhanced computed tomographic (CT) image, and early-and delayed-phase images, respectively. $A 4.2-\mathrm{cm}$ mass (adrenal adenoma) can be seen in the left adrenal gland on dynamic CT (arrows). Tumor attenuation value of pre-contrast (TApre), TAearly and TAdelay were 15, 76 and $60 \mathrm{HU}$, respectively. Absolute percentage washout (APW) was $26.2 \%$. The mass was not diagnosed as adrenal adenoma using TApre $\leq 10 \mathrm{HU}$ or $A P W \geq 51.4 \%$. The predictive formula made a correct diagnosis of adrenal adenoma. The probability of adrenal adenoma using the formula was 0.9317. 
the scan timing of early and delayed phases was the same although the injection rate was slightly different between the two $(2.5 v s .2 .4-3.3 \mathrm{ml} / \mathrm{s})$. We believe that this small difference did not greatly affect the measurement of washout rate. Thirdly, our predictive formula of AA was obtained based on 4-minute delayed scanning. The appropriate predictive formula might depend on the delayed scan timing.

In conclusion, the washout rate of AA was correlated with CCR and TApre. The diagnostic potential of dynamic CT for diagnosing AA may be improved by changing the threshold of washout rate based on TApre substituted for CCR.

\section{Conflicts of Interest}

The Authors declare that they have no conflicts of interest in regard to this study. No Grant or Fund supported this work.

\section{Acknowledgements}

The Authors thank Professor Yoshinao Oda, Department of Anatomic Pathology, Kyushu University, for providing the pathological information for this article. We also thank Professor Masatoshi Eto, Department of Urology, Kyushu University, for providing the clinical information for this article.

\section{References}

1 Elsayes KM, Mukundan G, Narra VR, Lewis JS Jr., Shirkhoda A, Farooki A and Brown JJ: Adrenal masses: MR imaging features with pathologic correlation. Radiographics 24(Suppl 1): S73-86, 2004

2 Park BK, Kim B, Ko K, Jeong SY and Kwon GY: Adrenal masses falsely diagnosed as adenomas on unenhanced and delayed contrast-enhanced computed tomography: Pathological correlation. Eur Radiol 16: 642-647, 2006.

3 Yamada T, Ishibashi T, Saito H, Matsuhashi T, Majima K, Tsuda M, Takahashi S and Moriya T: Adrenal adenomas: relationship between histologic lipid-rich cells and $\mathrm{CT}$ attenuation number. Eur J Radiol 48: 198-202, 2003.

4 Korobkin M, Brodeur FJ, Francis IR, Quint LE, Dunnick NR and Londy F: CT time-attenuation washout curves of adrenal adenomas and nonadenomas. Am J Roentgenol 170: 747-752, 1998.

5 Israel GM, Korobkin M, Wang C, Hecht EN and Krinsky GA: Comparison of unenhanced CT and chemical shift MRI in evaluating lipid-rich adrenal adenomas. Am J Roentgenol 183: 215-219, 2004.

6 Szolar DH and Kammerhuber FH: Adrenal adenomas and nonadenomas: Assessment of washout at delayed contrastenhanced CT: Radiology 207: 369-375, 1998.

7 Caoili EM, Korobkin M, Francis IR, Cohan RH, Platt JF, Dunnick NR and Raghupathi KI: Adrenal masses: Characterization with combined unenhanced and delayed enhanced CT: Radiology 222: 629-633, 2002.
8 Kamiyama T, Fukukura Y, Yoneyama T, Takumi K and Nakajo $\mathrm{M}$ : Distinguishing adrenal adenomas from nonadenomas: Combined use of diagnostic parameters of unenhanced and short 5-minute dynamic enhanced CT protocol. Radiology 250: 474481, 2009.

9 Patel J, Davenport MS, Cohan RH and Caoili EM: Can established CT attenuation and washout criteria for adrenal adenoma accurately exclude pheochromocytoma? Am J Roentgenol 201: 1411-141, 2013.

10 Caoili EM, Korobkin M, Francis IR, Cohan RH and Dunnick NR: Delayed enhanced CT of lipid-poor adrenal adenomas. Am J Roentgenol 175: 1411-1415, 2000.

11 Nishie A, Asayama Y, Ishigami K, Kakihara D, Nakayama T, Ushijima Y, Takayama Y, Yokomizo A, Tatsugami K, Inokuchi J, Fujita N, Kubo Y, Aishima S, Hirakawa M and Honda H: Pathological manifestation of difference in washout pattern of adrenal hyperplasia on dynamic CT: J Med Imaging Radiat Oncol 58: 559-564, 2014.

12 Heinz-Peer G and Honigschnabl S, Schneider B, Niederle B, Kaserer $\mathrm{K}$ and Lechner $\mathrm{G}$ : Characterization of adrenal masses using MR imaging with histopathologic correlation. Am J Roentgenol 173: 15-22, 1999.

13 Boland GW, Blake MA, Hahn PF and Mayo-Smith WW: Incidental adrenal lesions: principles, techniques, and algorithms for imaging characterization. Radiology 249: 756-775, 2008.

14 Bilbey JH, McLoughlin RF, Kurkjian PS, Wilkins GE, Chan NH, Schmidt N and Singer J: MR imaging of adrenal masses: value of chemical-shift imaging for distinguishing adenomas from other tumors. Am J Roentgenol 164: 637-642, 1995.

15 Tsushima Y, Ishizaka $H$ and Matsumoto M: Adrenal masses: differentiation with chemical shift, fast low-angle shot MR imaging. Radiology 186: 705-709, 1993.

16 Park BK, Kim CK, Kim B and Lee JH: Comparison of delayed enhanced CT and chemical shift MR for evaluating hyperattenuating incidental adrenal masses. Radiology 243: 760-765, 2007.

17 Szolar DH, Korobkin M, Reittner P, Berghold A, Bauernhofer T, Trummer H, Schoellnast H, Preidler KW and Samonigg H: Adrenocortical carcinoma and adrenal pheochromocytomas: mass and enhancement loss evaluation at delayed contrastenhanced CT: Radiology 234: 479-485, 2005.

18 Erickson LA: Endocrine pathology, III: Adrenal gland. In: Essentials of Anatomic Pathology, Third Edition. Chen L and Bostwick DG (eds) Berlin, Springer, pp. 935-950, 2004.

19 Park BK, Kim CK and Kim B: Adrenal incidentaloma detected on triphasic helical CT: Evaluation with modified relative percentage of enhancement washout values. Br J Radiol 81: 526$530,2008$.
Received May 27, 2018

Revised June 6, 2018

Accepted June 11, 2018 